\title{
Vorstellung und studentische Evaluation digitaler Lernformate in zwei methodischen Grundlagenfächern der Mechanik
}

\author{
D. Bernstein, M. Schuster, M. Beitelschmidt \\ Professur für Dynamik und Mechanismentechnik, Institut für Festkörpermechanik, Fakultät Maschinenwesen, TU \\ Dresden
}

Die pandemiebedingte Umstellung der Präsenzlehre auf digitale Lernformate brachte vor allem für die Studierenden große Herausforderungen mit sich. Die beiden vorgestellten, methodischen Grundlagenfächer der Mechanik wurden vollständig digital angeboten. Zur Stoffvermittlung wurden aufgezeichnete Lehrvideos und zur Fragenbeantwortung vor allem Übungsforen eingesetzt. Mithilfe von Umfragen unter allen Prüfungsteilnehmenden wurden die eingesetzten Lernformate evaluiert. Es zeigt sich dabei, dass aufgezeichnete Vorlesungsvideos gegenüber Präsenzvorlesungen von den Studierenden als hilfreicher wahrgenommen werden. Als Vorteil wird dabei die Möglichkeit zum Anhalten und Wiederholen der Vorlesungsvideos genannt. Das Übungsforum wird zwar als eher hilfreich bewertet, jedoch nicht als adäquater Ersatz zur Präsenzübung eingeschätzt. Laut den studentischen Kommentaren spielt die direkte und unmittelbare Kommunikation in der Präsenzübung eine wichtige Rolle, die in einem Forum nicht erreicht werden kann. Die beste Bewertung unter den Lernformen erzielten kurze Einführungsvideos in die Thematik und Aufgaben der Übungen.

The pandemic-induced migration of classroom teaching to digital learning formats brought great challenges, especially for the students. The two presented methodical subjects in basic mechanics were offered completely digitally. Recorded teaching videos were used to convey the course content and mainly exercise forums were used to answer questions. The learning formats used were evaluated with the help of surveys among all examination participants. The results show that the students prefer recorded lecture videos over classroom lectures. The possibility of pausing and repeating the lecture videos was named as an advantage. The exercise forum is rated as helpful, but not as an adequate substitute for the classroom exercise. According to the student comments, direct and immediate communication plays an important role in the classroom exercise, which cannot be achieved in a forum. The best rating among the teaching formats was achieved by short introduction videos on the topic and tasks of the exercises.

*Corresponding author: david.bernstein@tu-dresden.de 


\section{Einführung}

Die durch die Corona-Pandemie bedingte Verlagerung des universitären Lehrbetriebs über mehrere Semester hinweg vom Hörsaal in den digitalen Raum hat vor allem für die Studierenden große Herausforderungen mit sich gebracht. Insbesondere in rechenintensiven Fächern wie der Technischen Mechanik ist die Vermittlung grundlegender Methoden in Präsenzübungen ein wesentlicher Bestandteil des normalen Kursprogramms. Dieser Artikel beschäftigt sich mit der Umsetzung der digitalen Lehre in zwei Fächern der Professur für Dynamik und Mechanismentechnik an der TU Dresden im Sommersemester 2020 und der Bewertung der umgesetzten Lernformate durch alle Studierenden, die an der entsprechenden schriftlichen Prüfung teilgenommen haben.

\section{Vorstellung der Lehrveranstaltungen}

Die Lehrveranstaltung Technische Mechanik Kinematik und Kinetik (TMKK) ist eine Pflichtveranstaltung mit 3 Semesterwochenstunden (SWS) Vorlesung und 2 SWS Übung im Diplomund Bachelorstudiengang Maschinenbau im 4. Semester. In Präsenzsemestern finden die Übungen in Gruppen von 20 bis 60 Studierenden statt. Der Hörerkreis der englischsprachigen Lehrveranstaltung Kinematik und Kinetik der Mehrkörpersysteme (MKS) besteht vor allem aus Studierenden der Studiengänge Maschinenbau in der Vertiefungsrichtung Simulationsmethoden im Maschinenbau (MB-SIM), Mechatronik (MT) und Computational Modelling and Simulation (CMS). Die Studierenden nehmen regulär an 2 SWS Vorlesung und 2 SWS Übung teil. Darüber hinaus besuchen Studierende der Maschinenbau-Vertiefungsrichtung Verarbeitungs- und Textilmaschinenbau (MB-VTMB) mit jeweils 1 SWS Vorlesung und Übung regulär den halben Kursinhalt. Es handelt sich für die Diplomstudiengänge (MBSIM, MB-VTMB, MT) um eine Wahlpflichtveranstaltung im Hauptstudium.

\section{Digitale Lernformate in der TMKK}

In der Lehrveranstaltung TMKK standen Vorlesungsaufzeichnungen zur Verfügung, die von der AG Fernstudium der Fakultät Maschinenwesen an der TU Dresden 2015 im regulären Vorlesungsbetrieb im Hörsaal aufgezeichnet wurden. Die jeweiligen Vorlesungsvideos wurden in der entsprechenden Semesterwoche auf der sächsischen Lernplattform OPAL unter Nutzung des Videoservers MAGMA freigeschaltet. Der Vorlesungsstoff wird darin hauptsächlich unter Nutzung eines Tablet-Computers handschriftlich vermittelt (siehe Abbildung 1).

Für die jeweiligen Übungen wurden ausführliche Einführungsfolien, Hinweise zur Lösung der einzelnen Aufgaben und ausführliche Lösungen der Übungsaufgaben zur Verfügung gestellt. Die ersten beiden Übungseinführungen sind darüber hinaus auf Video aufgezeichnet worden, was aus Kapazitätsgründen des Lehrpersonals für die weiteren Einführungen nicht fortgeführt wurde. Fragen zu den Übungsaufgaben und zur Prüfungsvorbereitung konnten in einem OPAL-Forum gestellt werden. Für jede Übung wurde ein separates Forum eingerichtet. Es wurden ca. aller zwei Wochen Online-Konsultationen angeboten, wobei dieses Angebot lediglich von durchschnittlich 5 Studierenden genutzt wurde.

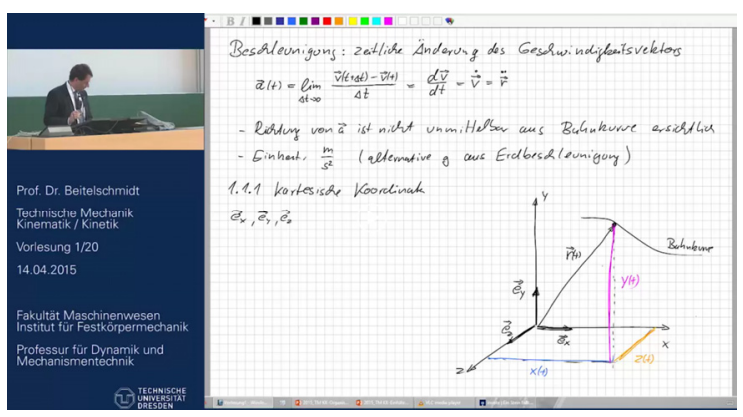

Abb. 1: Vorlesung TMKK, Aufnahme der AG Fernstudium der Fakultät Maschinenwesen an der TU Dresden.

\section{Digitale Lernformate in der MKS}

Die Vorlesungen der Lehrveranstaltung MKS sind als Wechsel aus Folien-Vortrag und handschriftlichem Tablet-Vortrag konzipiert. Die Vorlesungsvideos (siehe Abbildung 2) wurden während des Semesters aufgezeichnet. Die tatsächliche Videodauer ist aufgrund fehlender Rückfragen und spontaner zusätzlicher Erläuterungen kürzer als die übliche Vorlesungszeit von 90 Minuten. 


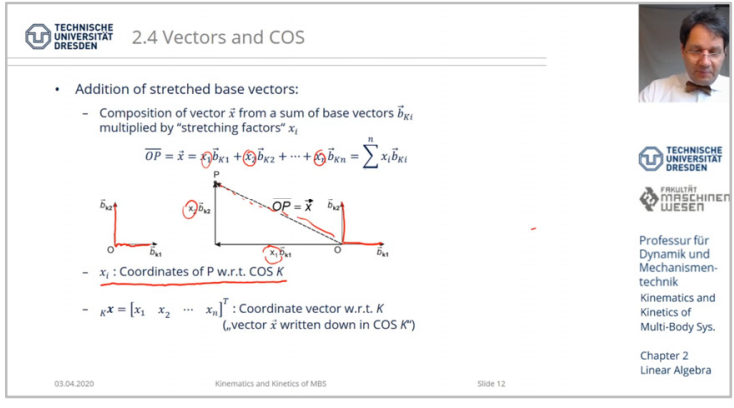

Abb. 2: Vorlesung MKS, handschriftlich ergänzte Folie, Aufnahme mit OBS Studio.

Im Zuge der Umstellung auf eine englischsprachige Veranstaltung wurden im Vorjahr folienbasierte, ca. zehnminütige Einführungsvideos (Intro-Videos) für alle Übungen produziert (siehe Abbildung 3). Zur Beantwortung von Fragen zu den Übungen und Musterklausuren wurde wie in der TMKK das OPAL-Forum genutzt.

Den Abschluss der Lehrveranstaltung bildet ein Simulationskomplex, der im digitalen Semester teilweise in Präsenz stattfand, allerdings unter sehr geringer Teilnahme. Ziel des Simulationskomplex ist die Programmierung einer Mehrkörpersimulation in der kommerziellen Software MATLAB. In Vorbereitung auf die Prüfung fand eine Online- und eine Präsenz-Konsultation statt.

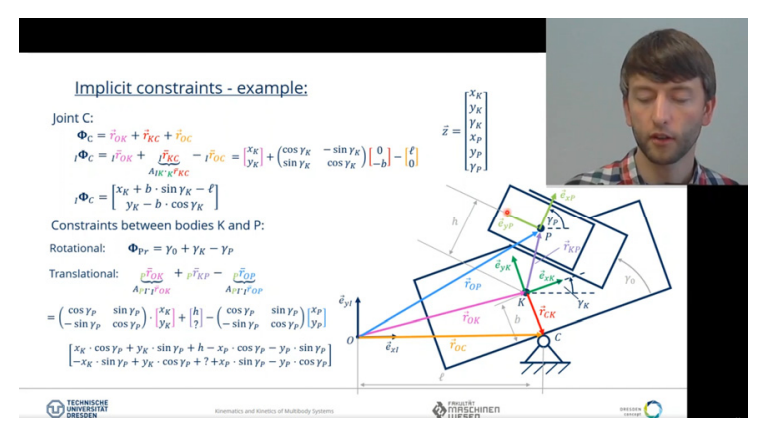

Abb. 3: Übungseinführung MKS, Beispielrechnung auf Powerpoint-Folie, Aufnahme mit OBS Studio

\section{Evaluation der Lehrveranstaltungen}

Zur Erhebung einer umfassenden Lehr-Evaluation wurde am Ende der jeweiligen schriftlichen Präsenzprüfung eine anonyme Umfrage mit ca. 20 Fragen und Kommentarfeldern unter allen Prüfungsteilnehmern durchgeführt. So liegen für die Lehrveranstaltung TMKK 338 und für die MKS 70 ausgefüllte Umfragen vor. Aus dem Vorjahr liegen für die MKS weitere 52 ausgefüllte Umfragen mit teilweise identischer Fragestellung vor, was einen Vergleich mit dem Präsenzsemester erlaubt.

In den Diagrammen zur Veranstaltung TMKK ist der Anteil der Studierenden orange gekennzeichnet, die die Prüfung wiederholt geschrieben haben und damit die Veranstaltung erstmalig bereits in einem regulären Präsenzsemester besucht haben. Dabei handelt es sich um $16 \%$ der Prüfungsteilnehmenden. In der Veranstaltung MKS wird in die Studierendengruppen Maschinenbau-SIM/Mechatronik (blau), CMS (orange) und Maschinenbau-VTMB (gelb) unterschieden. Aufgrund vernachlässigbarer Durchfallquoten spielt hier der Anteil an Prüfungswiederholenden eine untergeordnete Rolle.

\begin{tabular}{|c|c|c|c|c|c|c|}
\hline \multirow{2}{*}{$\begin{array}{ll}\text { Prüfungsversuch: } & \circ \text { Erstversuch } \\
\text { Muttersprache: } & \circ \text { Deutsch }\end{array}$} & \multicolumn{6}{|c|}{ O Zweit- oder Drittversuch } \\
\hline & & & & & & \\
\hline \begin{tabular}{|l}
$\begin{array}{l}\text { Aussage } \\
\text { (Bitte bewerten Sie nur Aussagen, zu denen Sie aussage- } \\
\text { tahig gind) }\end{array}$ \\
\end{tabular} & $\begin{array}{c}\text { Triftr } \\
\text { voll zu }\end{array}$ & \begin{tabular}{|l} 
Trifft zu \\
un
\end{tabular} & $\begin{array}{c}\text { Teils/ } \\
\text { Teils }\end{array}$ & \begin{tabular}{|c|c|} 
Triftr \\
kaum zu
\end{tabular} & \begin{tabular}{|c|} 
Trifitt \\
nicht zu
\end{tabular} & $\begin{array}{c}\text { Keine } \\
\text { Aussage } \\
\text { mogglich }\end{array}$ \\
\hline $\begin{array}{l}\text { Ich konnte der Vorlesung inhaltich sehr gut } \\
\text { folgen. }\end{array}$ & 0 & $\circ$ & $\circ$ & $\circ$ & $\circ$ & $\circ$ \\
\hline $\begin{array}{l}\text { Ich konnte den Video-Vorlesungen besser fol- } \\
\text { gen, als „normalen" Vorlesungen in Präsenz. }\end{array}$ & $\circ$ & $\circ$ & $\circ$ & 0 & $\circ$ & $\circ$ \\
\hline $\begin{array}{l}\text { Ich habe die Videos zur Vorlesung in der je- } \\
\text { weils vorgesesehenen Woche angeschaut. }\end{array}$ & $\circ$ & $\circ$ & $\circ$ & $\circ$ & $\circ$ & $\circ$ \\
\hline $\begin{array}{l}\text { Ich haste technische Probleme, die Videos an- } \\
\text { zuschauen. }\end{array}$ & 0 & $\circ$ & $\circ$ & $\circ$ & $\circ$ & $\circ$ \\
\hline $\begin{array}{l}\text { Die Einführungen und Lösungen zu den Übun- } \\
\text { gen waren sehr hilfreich für mich. }\end{array}$ & 0 & $\circ$ & 0 & 0 & $\circ$ & 0 \\
\hline $\begin{array}{l}\text { Ich habe die Ubbungsaufgaben in der jeweils } \\
\text { vorgesehenenen Woche bearbeitet. }\end{array}$ & 0 & $\circ$ & $\circ$ & $\circ$ & $\circ$ & $\circ$ \\
\hline $\begin{array}{l}\text { lch habe mich regelmäßig mit Kommilitonen } \\
\text { uber Vorlesung bzw. Ubung ausgetauscht. }\end{array}$ & 0 & $\circ$ & 0 & 0 & $\circ$ & $\circ$ \\
\hline $\begin{array}{l}\text { Das Übungsforum war für mich sehn hilfreich } \\
\text { zur Bearbeitung der Übungsaufgaben. }\end{array}$ & 0 & $\circ$ & $\circ$ & 0 & $\circ$ & 0 \\
\hline $\begin{array}{l}\text { Ich hatte technische Probleme bei der Teil- } \\
\text { nahme an den Online-Konsultationen. }\end{array}$ & $\circ$ & $\circ$ & $\circ$ & $\circ$ & 0 & $\circ$ \\
\hline $\begin{array}{l}\text { Das Prüfungsforum hat mir in der Prüfungs- } \\
\text { vorbereitung sehr geholfen. }\end{array}$ & $\circ$ & $\circ$ & 0 & 0 & $\circ$ & $\circ$ \\
\hline $\begin{array}{l}\text { Vor der Prüfung habe ich mich sehr gut vorbe- } \\
\text { reitet gefühlt. }\end{array}$ & $\circ$ & $\circ$ & $\circ$ & 0 & $\circ$ & $\circ$ \\
\hline $\begin{array}{l}\text { Bei der Prüunng habe ich gemerkt, dass ich } \\
\text { sehr gut vorbereitet war. }\end{array}$ & 0 & $\circ$ & $\circ$ & 0 & $\circ$ & $\circ$ \\
\hline $\begin{array}{l}\text { Die Möglichkeit, das Prüfungsergebnis im } \\
\text { Nachgang zu annullieren, beeinflusste meine } \\
\text { Prứfungsvorbereitung. }\end{array}$ & 0 & $\circ$ & $\circ$ & $\circ$ & $\circ$ & $\circ$ \\
\hline $\begin{array}{l}\text { Es fiel mir in diesem Semester schwer, mich } \\
\text { für die Bearbeitung der Kursinhalte zu motivie- } \\
\text { ren. }\end{array}$ & $\circ$ & $\circ$ & $\circ$ & 0 & $\circ$ & $\circ$ \\
\hline
\end{tabular}

Abb. 4: Vorderseite des Umfragebogens in der TMKK

Wie in Abbildung 4 dargestellt, boten die meisten Fragen der Erhebung 5 Antwortmöglichkeiten von "1: Trifft voll zu“ (links) über "3: Teils/Teils“ (Mitte) bis "5: Trifft nicht zu“ (rechts). Abweichende Antwortmöglichkeiten sind in den jeweiligen Diagrammen gekennzeichnet.

\section{Vergleich von Präsenz- und Online-Se- mester}

Beim Vergleich zwischen Präsenz- und Onlinesemester ergibt sich ein differenziertes Bild. Abbildung 5 zeigt die Bewertung der Aussage 
durch die Studierenden der TMKK, ob sie der Online-Vorlesung besser folgen konnten als normalen Vorlesungen in Präsenz. Mit einer durchschnittlichen Bewertung von 2,55 geben die Studierenden an, einer Online-Vorlesung besser folgen zu können. Die Aussage, ob sie der Online-Vorlesung sehr gut folgen konnten, wurde durchschnittlich mit 2,41 bewertet.

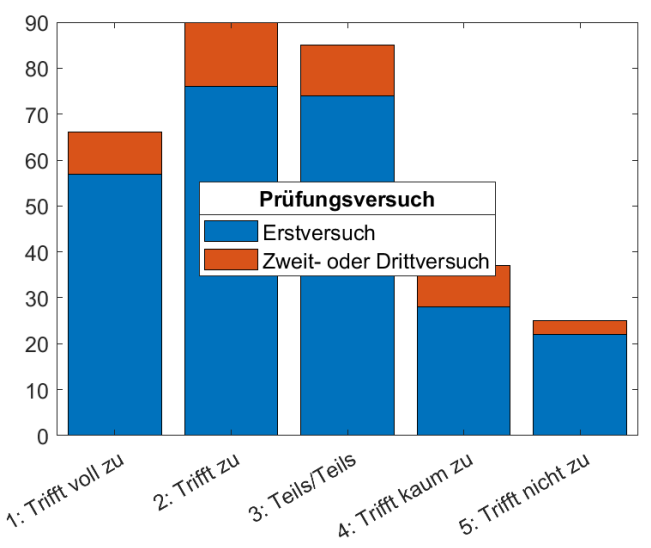

Abb. 5: "Ich konnte den Video-Vorlesungen besser folgen als normalen Vorlesungen in Präsenz." (TMKK)

Dies bestätigt auch der direkte Vergleich der Vorlesung MKS zwischen den Sommersemestern 2019 und 2020 in Abbildung 6. Auf die Frage, als wie hilfreich die jeweilige Vorlesung empfunden wurde, schneidet die Online-Vorlesung mit einer durchschnittlichen Bewertung von 2,44 besser ab als die Präsenzvorlesung des Vorjahres mit einer durchschnittlichen Bewertung von 2,74 .

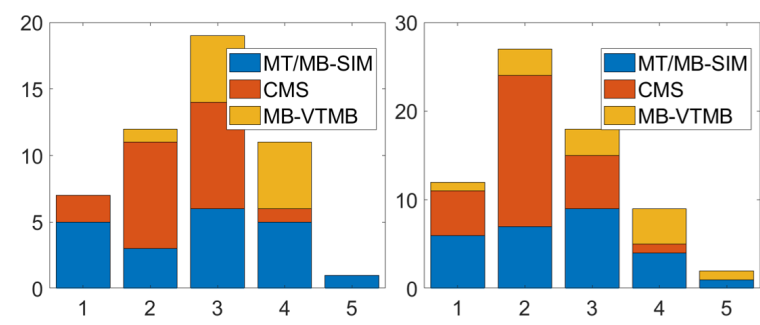

Abb. 6: "Die Vorlesung war sehr hilfreich für mich" (MKS). Links: Präsenz 2019; Rechts: Digital 2020

Eine mögliche Begründung für das bessere Abschneiden der Online-Vorlesung kann den handschriftlichen Kommentaren in der Umfrage entnommen werden. Es wurde hier mehrfach die Möglichkeit des Anhaltens und Wiederholens der Videovorlesung positiv hervorgehoben. Ein Studierender konkretisierte es dahingehend, dass das Zuhören und Schreiben durch die Pause-Funktion separat voneinander erfolgen kann. Ein anderer betont die Wiederholbarkeit "schwieriger Stellen" in der Videovorlesung.

Es sei auf die Besonderheit der betrachteten Veranstaltungen hingewiesen, dass in den Vorlesungen häufig komplexe Herleitungen und Rechenwege vorgeführt werden. Auf die individuelle Lerngeschwindigkeit des einzelnen Studierenden kann dabei in der Präsenzvorlesung nur sehr begrenzt eingegangen werden.

Dass in der TMKK ungefähr die Hälfe der Studierenden mehr als 75\% der Vorlesungen angeschaut haben (siehe Abbildung 7), deckt sich mit den Teilnehmerzahlen der Vorlesung in Präsenzsemestern der Veranstaltung.

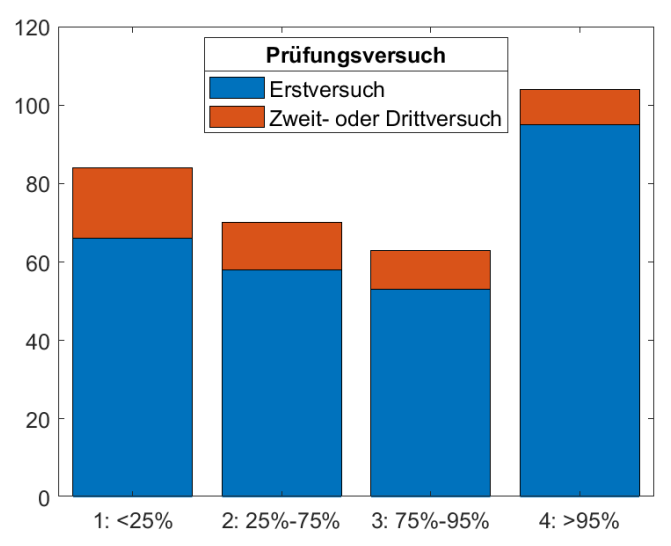

Abb. 7: Angeschaute Vorlesungsvideos. (TMKK)

Wie aus Abbildung 8 hervorgeht, schneidet allerdings der gesamte MKS-Kurs bestehend aus Vorlesung und Übung im Online-Semester schlechter $a b$ als im Präsenz-Semester. Die Aussage, ob der Kursinhalt im Online-Semester deutlich schlechter verständlich ist als im regulären Semester, wird in der MKS durchschnittlich mit 2,77 bewertet.

Die im Gegensatz dazu positive Bewertung der Vorlesung lässt darauf schließen, dass Übungen in Präsenz von Seiten der Studierenden ein deutliches Qualitätsmerkmal darstellen.

Ein großer Teil der Studierenden gibt an, zumindest teilweise Motivationsprobleme zur Bearbeitung der Kursinhalte gehabt zu haben (siehe Abbildung 9). Die Aussage, ob es den Studierenden schwerfiel, sich für die Kursinhalte zu motivieren, wird in der TMKK durchschnittlich mit 2,61 bewertet. 


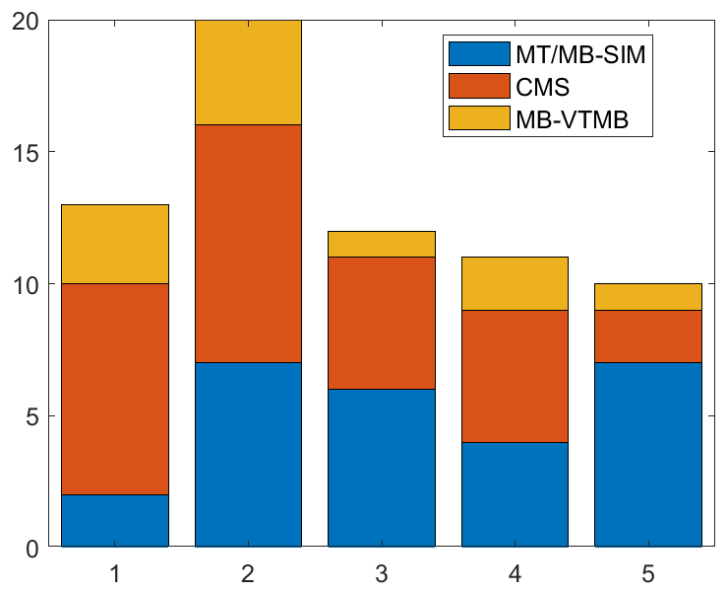

Abb. 8: „Der Kursinhalt ist in einem Online-Semester deutlich schlechter verständlich als in einem regulären." (MKS)

Dies spiegelt sich, wie in Abbildung 10 zu sehen ist, auch darin wider, dass der größere Teil der Studierenden der TMKK im Semester laut eigenen Angaben weniger als die wöchentlich geplante Präsenzzeit für den Kurs aufgewendet hat. Ein derartiges Ergebnis zeigte sich auch bei der Befragung der MKS-Studierenden.

In der MKS wird die Aussage, dass die Studierenden Probleme hatten, am Kursinhalt zeitlich dranzubleiben, mit durchschnittlich 3,10 bewertet. Von Studierenden der TMKK wird die Aussage, ob die Übungsaufgaben in der jeweils vorgesehenen Woche bearbeitet wurden, durchschnittlich mit 3,28 bewertet. Damit hatte der größere Teil der Studierenden zumindest teilweise Probleme, dem vorgegebenen Zeitplan im Online-Studium zu folgen. Da keine Vergleichsdaten aus dem Präsenzsemester vorliegen, kann hier kein solider Vergleich zwischen Online- und Präsenzsemester gezogen werden.

Die sehr geringe Teilnehmerzahl am Simulationskomplex in der MKS im Online-Semester ist ein weiteres Indiz für die verzögerte Bearbeitung des Kursinhaltes durch die Studierenden, da für eine erfolgreiche Bearbeitung des Simulationskomplexes die vollständige Bearbeitung der vorausgehenden Übungen notwendig ist.

In der TMKK wurde durch ein Kommentarfeld abgefragt, aus welchen Gründen nicht zeitlich am Kursinhalt drangeblieben wurde. Als Beispielantworten wurde "Motivation, Stress, Verständnisprobleme" angegeben.
Häufig wurde durch die Studierenden die fehlende Motivation, Verständnisprobleme und die fehlende Trennung zwischen Studium und Freizeit benannt. Für die Häufigkeit der ersten beiden Antworten spielen mutmaßlich die Beispielantworten eine Rolle.

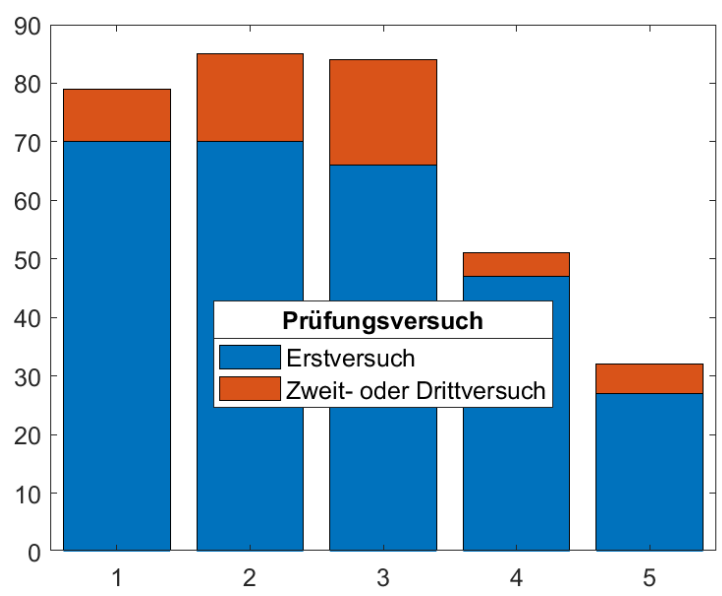

Abb. 9: „Es fiel mir in diesem Semester schwer, mich für die Bearbeitung der Kursinhalte zu motivieren." (TMKK)

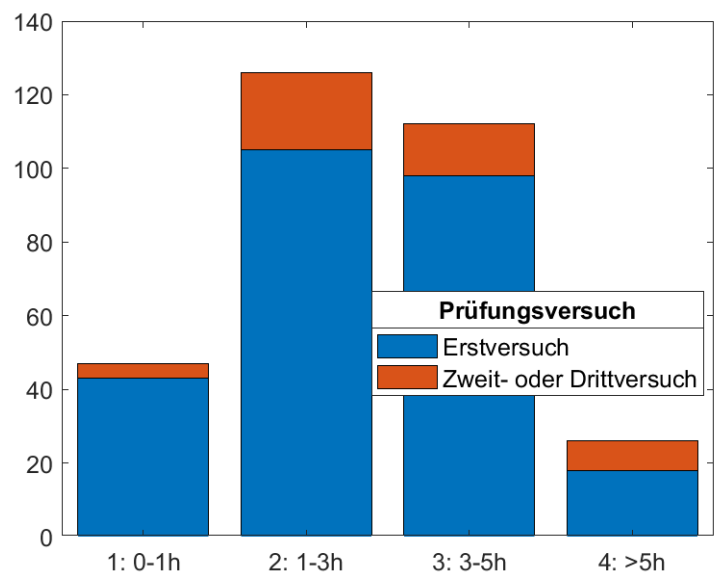

Abb. 10: Wöchentlicher Zeitaufwand für den Kurs. (TMKK, wöchentliche Präsenszeit wäre 3,75 h gewesen)

Aus Abbildung 11 lässt sich schließen, dass der kleinere Teil der Studierenden regelmäßigen, fachlichen Austausch mit Kommilitonen pflegt. Die Aussage, ob sich die Studierenden regelmäßig mit Kommilitonen über Kursinhalte ausgetauscht haben, wird in der TMKK durchschnittlich mit 3,36 bewertet. Der größere Teil der Studierenden erarbeitete sich die Kursinhalte demnach im Selbststudium, was im deutlichen Kontrast zum Präsenz-Übungsbetrieb steht. 


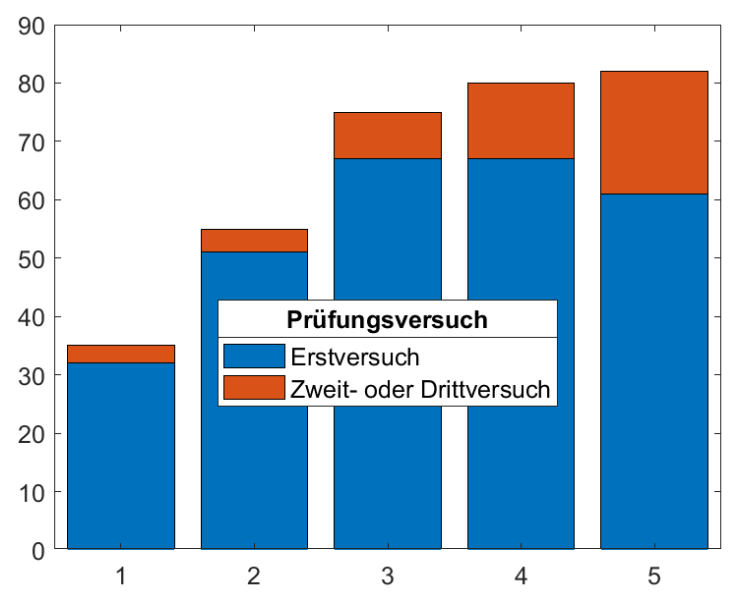

Abb. 11: "Ich habe mich regelmäßig mit Kommilitonen über die Vorlesung bzw. Übung ausgetauscht." (TMKK)

\section{Bewertung der einzelnen Lernformen im Übungsbetrieb}

Wie bereits erwähnt, sind in den vorgestellten Lehrveranstaltungen weitestgehend asynchrone Lernformate für den Übungsbetrieb gewählt worden.

Abbildung 12 zeigt dabei, dass das zur Verfügung gestellte, schriftliche Übungsmaterial, bestehend aus Einführungen und Musterlösungen in der TMKK als hilfreich wahrgenommen wurde. Die Aussage, ob das entsprechende Material sehr hilfreich war, wurde mit durchschnittlich 2,41 bewertet.

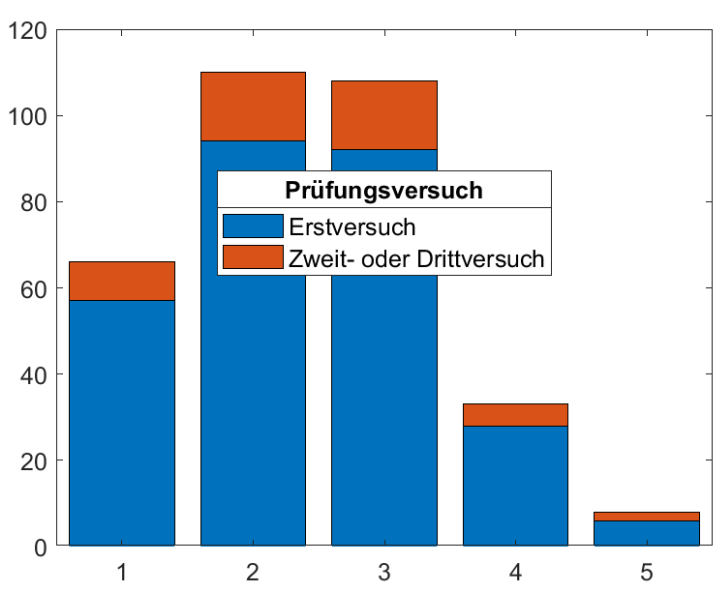

Abb. 12: "Die Einführungen und Lösungen zu den Übungen waren sehr hilfreich." (TMKK)

Etwas schlechter wurde die Hilfsmöglichkeit durch das Übungsforum bewertet, wie in Abbildung 13 dargestellt. Die Aussage, ob das
Übungsforum sehr hilfreich für die Studierenden war, wurde in der TMKK durchschnittlich mit 3,00 und in der MKS durchschnittlich mit 2,55 bewertet.

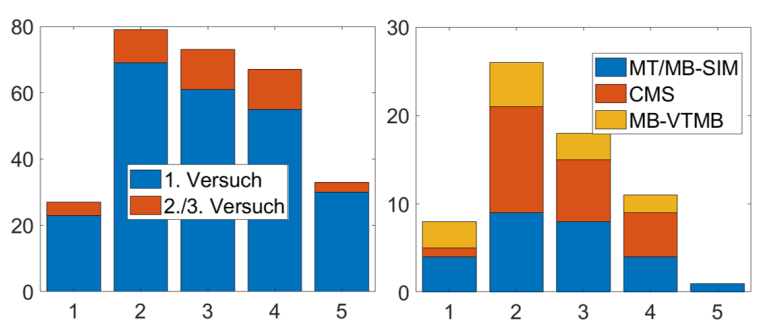

Abb. 13: „Das Übungsforum war für mich sehr hilfreich". Links: TMKK; Rechts: MKS

Die Kommentare zum Forum in der TMKK geben entsprechend ein gemischtes Bild ab. Einerseits wurden die geringe Übersichtlichkeit und die hohe Wartezeit auf Antwort auf eine gestellte Frage mehrfach bemängelt. Andererseits wurde mehrfach angegeben, lediglich die Forenbeiträge gelesen zu haben und daraus profitiert zu haben, ohne selbst Fragen zu stellen. Ein Kommentar merkte positiv an, dass man durch das Forum auf Probleme stieß, die man selbst nicht erkannt habe. Weiter wurde angemerkt, dass die Möglichkeit, anonyme Fragen zu stellen, die Hemmschwelle reduziere, das Forum aktiv zu nutzen. Sehr häufig wurde in den Kommentarfeldern der Wunsch nach Übungen in Präsenz angegeben, da die Fragenbeantwortung im Forum die direkte Kommunikation in einer Präsenzübung nicht ersetzen könnte.

Wie Abbildung 14 zu entnehmen ist, erzielten die kurzen Intro-Videos, die in der MKS zur Übungseinführung eingesetzt worden sind, die mit Abstand beste Bewertung. Die Frage, ob die Intro-Videos sehr hilfreich für die Studierenden waren, wurde in der MKS im Präsenzsemester 2019 durchschnittlich mit 1,58 und im Online-Semester 2020 durchschnittlich mit 1,64 bewertet.

Wie in Abbildung 15 dargestellt, wurden die kurzen Intro-Videos durch den größeren Teil der Studierenden mehrfach angeschaut, wohingegen die längeren Vorlesungsvideos von der Mehrzahl der Studierenden einmalig angeschaut wurden. 


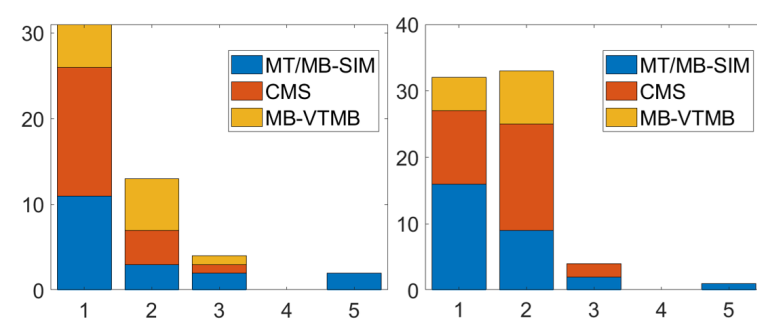

Abb. 14: „Die Intro-Videos waren sehr hilfreich für mich." (MKS) Links: 2019; Rechts: 2020

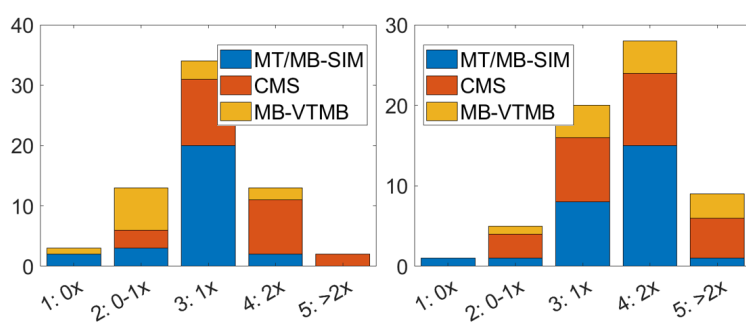

Abb. 15: "Wie oft haben Sie jedes einzelne Video durchschnittlich angeschaut?" Links: Vorlesungsvideo; Rechts: Intro-Video

Die Aussage, ob die Studierenden durch IntroVideos und Forum in der Lage waren, die Übungsaufgaben im Selbststudium zu lösen, wurde in der MKS im Online-Semester 2020 durchschnittlich mit 2,16 bewertet. Die ähnliche Aussage, ob die Studierenden (nur) durch die Intro-Videos dazu in der Lage waren, wurde in der MKS im Präsenz-Semester 2019 durchschnittlich mit 2,12 bewertet.

\section{Prüfungsvorbereitung}

Wie in Abbildung 16 dargestellt, fällt der gefühlte Vorbereitungsstand bei den Studierenden im digitalen Semester etwas schlechter aus als der im Präsenz-Semester. Die Aussage, ob sich die Studierenden sehr gut auf die Prüfung vorbereitet gefühlt haben, wird in der MKS im Präsenz-Semester 2019 durchschnittlich mit 2,62 und im Online-Semester 2020 durchschnittlich mit 2,91 bewertet.

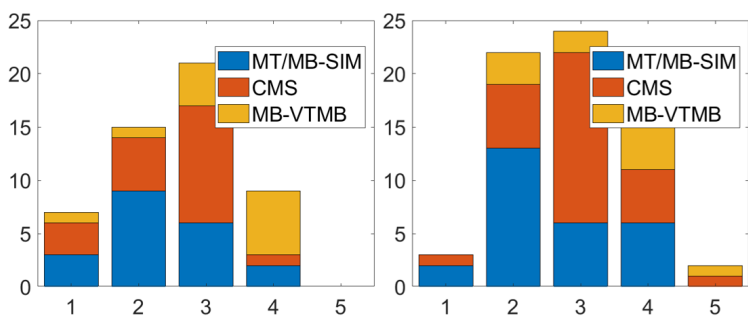

Abb. 16: „Vor der Prüfung habe ich mich sehr gut vorbereitet gefühlt." (MKS) Links: 2019; Rechts: 2020
An der Technischen Universität Dresden bestand im Sommersemester 2020 die Möglichkeit, Prüfungsleistungen im Nachgang unbegründet annullieren zu können. Wie aus Abbildung 17 hervorgeht, sieht sich der größere Teil der Studierenden durch diese Möglichkeit in geringem Maße in ihrer Prüfungsvorbereitung beeinflusst. Die Aussage, ob die Möglichkeit, das Prüfungsergebnis im Nachgang zu annullieren, die Prüfungsvorbereitung beeinflusste, wurde von den Studierenden in der MKS durchschnittlich mit 3,78 und in der TMKK durchschnittlich mit 3,89 bewertet.

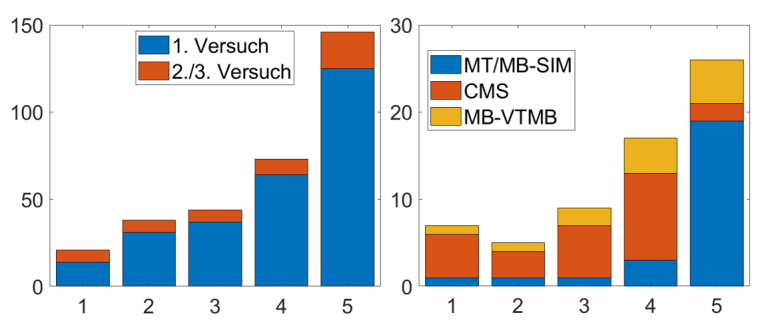

Abb. 17: „Die Möglichkeit, das Prüfungsergebnis im Nachgang zu annullieren, beeinflusste meine Prüfungsvorbereitung. "Links: TMKK; Rechts: MKS

\section{Zusammenfassung}

Es ergibt sich ein differenziertes Bild aus der Evaluation der beiden untersuchten Lehrveranstaltungen. So scheint eine Online-Vorlesung für viele Studierende Vorteile gegenüber einer Präsenzvorlesung mit sich zu bringen. Dahingegen war im digitalen Semester kein adäquater Ersatz für Präsenz-Übungen möglich. Das Angebot von Online-Konsultationen zur Übung wurde kaum wahrgenommen und das Übungsforum bietet aufgrund der zeitverzögerten Kommunikation keinen gleichwertigen Ersatz für eine direkte Kommunikation. Prägnante Video-Übungseinführungen werden hingegen als hilfreichstes Kurselement angesehen.

\section{Danksagung}

Ein großer Dank gilt Anja Jablonski und Falko Berger, die in besonderem Maße an der Auswertung der Umfragen mitgewirkt haben. 